FACTA UNIVERSITATIS (NIŠ)

Ser. Math. Inform. Vol. 34, No 3 (2019), 563-572

https://doi.org/10.22190/FUMI1903563A

\title{
ON THE EIGENVALUES OF N-CAYLEY GRAPHS: A SURVEY
}

\author{
Majid Arezoomand
}

(C) 2019 by University of Niš, Serbia | Creative Commons Licence: CC BY-NC-ND

Abstract. A graph $\Gamma$ is called an $n$-Cayley graph over a group $G$ if $\operatorname{Aut}(\Gamma)$ contains a semi-regular subgroup isomorphic to $G$ with $n$ orbits. In this paper, we review some recent results and future directions around the problem of computing the eigenvalues on $n$-Cayley graphs.

Keywords: $n$-Cayley graph; eigenvalues; semi-regular subgroup.

\section{Introduction}

The spectrum of a graph is one of the most important algebraic invariants as it is known that numerous proofs in graph theory depend on the spectrum of graphs. In particular, eigenvalues of Cayley graphs have attracted increasing attention due to their prominent roles in algebraic graph theory and applications in many areas such as expanders, chemical graph theory, quantum computing, etc [21]. This paper is a survey of the literature on the eigenvalues of graphs having a semi-regular of subgroup of their automorphism groups.

A digraph $\Gamma$ is a pair $(V, E)$ of vertices $V$ and edges $E$ where $E \subseteq V \times V$. A graph is a digraph with no edges of the form $(\alpha, \alpha)$ and with the property that $(\alpha, \beta) \in E$ implies $(\beta, \alpha) \in E$. The set of all permutations of $V$ which preserve the adjacency structure of $\Gamma$ is called the automorphism group of $\Gamma$; it is denoted by Aut $(\Gamma)$. In this paper all digraphs have no loops. For the most part our notation and terminology are standard and mainly taken from [9] (for graph theory) and [16] (for representation theory of finite groups). For the graph-theoretic and group-theoretic terminology not defined here we refer the reader to $[9,16]$.

Let $\Gamma$ be a (di)graph with $n$ vertices. The adjacency matrix of $A$ of $\Gamma$ is an $n \times n$ matrix with $i j$-entry equal to 1 if $i$ th and $j$ th vertices are adjacent and 0 otherwise. The spectrum of a graph is the multi-set of eigenvalues of its adjacency matrix. It

Received January 04, 2019; accepted March 15, 2019

2010 Mathematics Subject Classification. Primary 05C50; Secondary 05C25,05C31 
is known that numerous proofs in graph theory depend on the spectrum of graphs and the spectrum of a graph is one of the most important algebraic invariants [9].

Let $G$ be a group and $S$ be a subset of $G$ not containing the identity element of $G$. The Cayley (di)graph of $G$ with respect to $S$ is a graph with a vertex set $G$ where $(g, h)$ is an arc whenever $h g^{-1} \in S$. A large number of results on spectra of Cayley graphs have been produced over the last more than four decades. For a survey of the literature on eigenvalues of Cayley graphs and their applications see [21].

By a theorem of Sabidussi [26], a (di)graph $\Gamma$ is a Cayley graph over a group $G$ if $\operatorname{Aut}(\Gamma)$ contains a regular subgroup of $\operatorname{Aut}(\Gamma)$ isomorphic to $G$. As a generalization, a (di)graph $\Gamma$ is called an $n$-Cayley (di)graph over a group $G$ if there exists a semiregular subgroup of $\operatorname{Aut}(\Gamma)$ isomorphic to $G$ with $n$ orbits (of equal size). Since every regular subgroup is a transitive semi-regular subgroup, every Cayley (di)graphs is a 1-Cayley (di)graph. Also a Cayley graph over a finite group $G$ having a subgroup $H$ of index $n$ is an $n$-Cayley graph over $H$ [1, Lemma 8]. $n$-Cayley graphs over cyclic groups are called $n$-circulant. In particular 2-Cayley and 3-Cayley graphs over cyclic groups are called bicirculant and tricirculant graphs [24], respectively. Unlike Cayley graphs, in general $n$-Cayley graphs are not vertex-transitive for $n \geq 2$. Furthermore, there are vertex-transitive $n$-Cayley graphs which are not Cayley graphs such as generalized Petersen graphs. Undirected and loop-free 2-Cayley graphs are called, by some authors, semi-Cayley graphs $[25,3]$ and also bi-Cayley graphs [17]. In this paper, we follow [25] to use the term semi-Cayley.

$n$-Cayley graphs, in particular when $n=2$ or $n=3$, have played an important role in many classical fields of graph theory, such as strongly regular graphs $[19,22$, $23,24,25]$, automorphisms [2, 15, 28], isomorphisms [3, 5], symmetry properties of graphs $[10,11,20]$ and the spectrum of graphs $[1,4,8,12,13]$. In this paper, we review recent results and future directions of some problems related to the spectrum of $n$-Cayley graphs.

\section{Presentation of $n$-Cayley graphs}

Recall that a (di)graph $\Gamma$ is called an $n$-Cayley graph over a group $G$ if $\operatorname{Aut}(\Gamma)$ contains a semi-regular subgroup isomorphic to $G$ with $n$ orbits (of equal size). It is proved that every $n$-Cayley graph over a group $G$ can be presented by suitable $n^{2}$ subsets of $G$ :

Lemma 2.1. ([1, Lemma 2]) A digraph $\Gamma$ is $n$-Cayley digraph over $G$ if and only if there exist subsets $T_{i j}$ of $G$, where $1 \leq i, j \leq n$, such that $\Gamma$ is isomorphic to a digraph $X$ with vertex set $G \times\{1,2, \ldots, n\}$ and edge set

$$
E(X)=\bigcup_{1 \leq i, j \leq n}\left\{((g, i),(t g, j)) \mid g \in G \text { and } t \in T_{i j}\right\} .
$$

By Lemma 2.1, an $n$-Cayley (di)graph is characterized by a group $G$ and $n^{2}$ subsets $T_{i j}$ of $G$ (some subsets may be empty). So we denote an $n$-Cayley (di)graph 
with respect to $n^{2}$ subsets $T_{i j}$ by $\Gamma=\operatorname{Cay}\left(G ; T_{i j} \mid 1 \leq i, j \leq n\right)$. It is easy to see that $\operatorname{Cay}\left(G ; T_{i j} \mid 1 \leq i, j \leq n\right)$ is undirected if and only if $T_{i j}^{-1}=T_{j i}$ for all $1 \leq i, j \leq n$. Also it is loop-free if $1 \notin T_{i i}$ for all $1 \leq i \leq n$. Let $\Gamma$ be a 2-Cayley graph which is undirected and loop-free. Then there exist three subsets $R=T_{11}$, $L=T_{22}, S=T_{12}$ and $T_{21}=S^{-1}$ of $G$ such that $R=R^{-1}, L=L^{-1}$ and $1 \notin R \cup L$ and $\Gamma=\operatorname{Cay}\left(G ; T_{i j} \mid 1 \leq i, j \leq 2\right)$. We denote this graph with $\operatorname{SC}(G ; R, L, S)$ and call it semi-Cayley graph. In the case $R=L=\varnothing$, we denote it by BCay $(G ; S)$ and call it bi-Cayley graph.

There are a lot of examples of $n$-Cayley graphs, $n \geq 2$. Here we provide some.

Example 2.1. Let $P$ be the Petersen graph. Then $P=\operatorname{SC}(G ; R, L, S)$, where $G=\langle a\rangle \cong$ $\mathbb{Z}_{5}, R=\left\{a, a^{4}\right\}, L=\left\{a^{2}, a^{3}\right\}$ and $S=\{1\}$.

Example 2.2. ([1, Lemma 8]) Let $\Gamma=\operatorname{Cay}(G, S)$ be a Cayley (di)graph. Suppose that there exists a subgroup $H$ of $G$ with index $n$. If $\left\{t_{1}, \ldots, t_{n}\right\}$ is a left transversal to $H$ in $G$, then $\Gamma \cong \operatorname{Cay}\left(H, T_{i j} \mid 1 \leq i, j \leq n\right)$, where $T_{i j}=\left\{h \in H \mid t_{j}^{-1} h t_{i} \in S\right\}=H \cap t_{j} S t_{i}^{-1}$.

Example 2.3. The $I$-graph $I(n, j, k)$ is a cubic graph of order $2 n$ with vertex set $\left\{u_{i}, v_{i} \mid\right.$ $0 \leq i \leq n-1\}$ and edge set $\left\{u_{i} u_{i+j}, u_{i} v_{i}, v_{i} v_{i+k}\right\}$. Graphs $I(n, 1, k)$ are called generalized Petersen graphs. It is easy to see that $I(n, j, k)=\operatorname{SC}(G ; R, L, S)$, where $G=\langle a\rangle \cong \mathbb{Z}_{n}$, $R=\left\{a^{j}, a^{-j}\right\}, L=\left\{a^{k}, a^{-k}\right\}$ and $S=\{1\}$.

Example 2.4. Let $R W(n, j, k)$ be a Rose Window graph, for the definition of graph see [18]. $R W(n, j, k)$ is a 4 -valent bicirculant graph isomorphic to $\operatorname{SC}(G ; R, L, S)$, where $G=\langle a\rangle \cong \mathbb{Z}_{n}, R=\left\{a, a^{-1}\right\}, L=\left\{a^{j}, a^{-j}\right\}$ and $S=\left\{1, a^{k}\right\}$.

Example 2.5. For given natural numbers $n \geq 3$ and $1 \leq r, j, k \leq n-1$, with $j \neq n / 2$ and $r \neq k$, the Tabačjn graph $T(n, r, k, j)$ is a pentavalent graph with vertex set $\left\{x_{i} \mid i \in\right.$ $\left.\mathbb{Z}_{n}\right\} \cup\left\{y_{i} \mid i \in \mathbb{Z}_{n}\right\}$ and edge set

$$
\left\{x_{i} x_{i+1} \mid i \in \mathbb{Z}_{n}\right\} \cup\left\{y_{i} y_{i+j} \mid i \in \mathbb{Z}_{n}\right\} \cup\left\{x_{i} y_{i+r} \mid i \in \mathbb{Z}_{n}\right\} \cup\left\{x_{i} y_{i+k} \mid i \in \mathbb{Z}_{n}\right\} .
$$

It is easy to see that $T(n, r, k, j)=\Gamma \cong \operatorname{SC}(G ; R, L, S)$, where $G=\langle a\rangle \cong \mathbb{Z}_{n}, R=\left\{a, a^{-1}\right\}$, $L=\left\{a^{j}, a^{-j}\right\}$ and $S=\left\{1, a^{r}, a^{k}\right\}$.

Example 2.6. Let $K_{r, r, \ldots, r}$ be the $n$-partite complete graph. Then $K_{r, r, \ldots, r}=\operatorname{Cay}\left(G ; T_{i j}\right.$ $1 \leq i, j \leq n)$, where $G$ is a finite group of order $r$, and for all $1 \leq i, j \leq n$ where $j \neq i$, $T_{i i}=\varnothing$ and $T_{i j}=G$.

\section{Eigenvalues of $n$-Cayley (di)graphs}

In 2007, the spectrum of bi-Cayley graphs over finite abelian groups computed in [29]:

Theorem 3.1. Let $\Gamma=\operatorname{BCay}(G, S)$ be a bi-Cayley graph over finite abelian group $G=\mathbb{Z}_{n_{1}} \times \ldots \times \mathbb{Z}_{n_{t}}$ with respect to $S$. Then eigenvalues of $\Gamma$ are

$$
\pm \sum_{\left(i_{1}, \ldots, i_{t}\right) \in S} \omega_{n_{1}}^{r_{1} i_{1}} \ldots \omega_{n_{t}}^{r_{t} i_{t}} \mid, \quad r_{j}=0, \ldots, n_{j}-1, \quad j=1, \ldots, t .
$$


In 2010, Gao and Luo improved Theorem 3.1. They studied the spectrum of semi-Cayley graphs over finite abelian groups. Using matrix theory, they derived a formula of the spectrum of semi-Cayley graphs over finite abelian groups:

Theorem 3.2. ([12, Theorem 3.2]) Let $\Gamma=\mathrm{SC}(G ; R, L, S)$ be a semi-Cayley graph over a finite abelian group $G=\mathbb{Z}_{n_{1}} \times \ldots \times \mathbb{Z}_{n_{t}}$. Then $\Gamma$ has eigenvalues

$$
\frac{\lambda_{r_{1} \ldots r_{t}}^{R}+\lambda_{r_{1} \ldots r_{t}}^{R} \pm \sqrt{\left(\lambda_{r_{1} \ldots r_{t}}^{R}-\lambda_{r_{1} \ldots r_{t}}^{L}\right)^{2}+4\left|\lambda_{r_{1} \ldots r_{t}}^{S}\right|^{2}}}{2},
$$

$r_{j}=0, \ldots, n_{j}-1, j=1, \ldots, t$, where $\lambda_{r_{1} \ldots r_{t}}^{X}=\sum_{\left(i_{1}, \ldots, i_{t}\right) \in X} \omega_{n_{1}}^{r_{1} i_{1}} \ldots \omega_{n_{t}}^{r_{t} i_{t}}$ and $\omega_{n}$ is the primitive nth root of unity.

Also the spectrum of a bi-Cayley graph of an arbitrary group with respects to a normal subset computed in [6, Theorem 2.1], a generalization of Theorem 3.1. In 2013, Theorem 3.2 extended to $n$-Cayley graphs, $n \geq 2$, over arbitrary groups by Arezoomand and Taeri in [1] using representation theory of finite groups. Let us recall some definitions of the latter paper. Let $G$ be a finite group and $\mathbb{C}[G]$ be the complex vector space of dimension $|G|$ with basis $\left\{e_{g} \mid g \in G\right\}$. We identify $\mathbb{C}[G]$ with the vector space of all complex-valued functions on $G$. Thus a function $\varphi: G \rightarrow \mathbb{C}$ corresponds to the vector $\varphi=\sum_{g \in G} \varphi(g) e_{g}$ and vice versa. In particular, the vector $e_{g}$, where $g \in G$, of the standard basis corresponds to the function $e_{g}$, where

$$
e_{g}(h)= \begin{cases}1 & h=g \\ 0 & h \neq g\end{cases}
$$

The (left) regular representation $\rho_{\text {reg }}$ of $G$ on $\mathbb{C}[G]$ is defined by its action on the basis $\left\{e_{h} \mid h \in G\right\}$; that is for all $g, h \in G, \rho_{\text {reg }}(g) e_{h}=e_{g h}$. Let $\operatorname{Irr}(G)=$ $\left\{\rho_{1}, \ldots, \rho_{m}\right\}$ be the set of all irreducible inequivalent $\mathbb{C}$-representations of $G$ and $d_{k}$ be the degree of $\rho_{k}, k=1, \ldots, m$. Let $e_{g}^{i}$ be the $1 \times n|G|$ vector with $n$ blocks, where $i$ th block is $e_{g}$, as defined, and other blocks are $0_{1 \times|G|}$ vectors. Let $V$ be the vector space with basis $\left\{e_{g}^{i} \mid g \in G, 1 \leq i \leq n\right\}$. Clearly $V \cong \underbrace{\mathbb{C}[G] \oplus \mathbb{C}[G] \oplus \cdots \oplus \mathbb{C}[G]}_{n-\text { times }}$, as $\mathbb{C}[G]=\left\langle e_{g} \mid g \in G\right\rangle$. So $\operatorname{dim}_{\mathbb{C}} V=n \operatorname{dim}_{\mathbb{C}} \mathbb{C}[G]=n|G|$. Let $\Gamma=\operatorname{Cay}\left(G ; T_{i j} \mid 1 \leq\right.$ $i, j \leq n)$ and $A=\left[a_{(g, i)(h, j)}\right]_{g, h \in G, 1 \leq i, j \leq n}$ be the adjacency matrix of $\Gamma$. Viewing $\bar{A}$ as the linear map

$$
\begin{aligned}
A: V & \rightarrow V \\
e_{g}^{i} & \mapsto \sum_{j=1}^{n} \sum_{h \in G} a_{(h, j)(g, i)} e_{h}^{j}, \quad 1 \leq i \leq n, g \in G,
\end{aligned}
$$

it is proved that:

Theorem 3.3. ([1, Theorem 6]) Let $\Gamma=\operatorname{Cay}\left(G ; T_{i j} \mid 1 \leq i, j \leq n\right)$ be an $n$-Cayley digraph over a finite group $G$ and $\operatorname{Irr}(G)=\left\{\rho_{1}, \ldots, \rho_{m}\right\}$. For each $l \in\{1, \ldots, m\}$, 
we define $n d_{l} \times n d_{l}$ block matrix $A_{l}:=\left[A_{i j}^{(l)}\right]$, where $A_{i j}^{(l)}=\sum_{t \in T_{j i}} \rho^{(l)}(t)$. Let $\chi_{A_{l}}(\lambda)$ and $\chi_{A}(\lambda)$ be the characteristic polynomial of $A_{l}$ and $A$, respectively. Then $\chi_{A}(\lambda)=\Pi_{l=1}^{m} \chi_{A_{l}}(\lambda)^{d_{l}}$.

Example 3.1. ([7, Corollary 2.3]) The eigenvalues of $I(n, j, k)$ are

$$
\cos (2 l j \pi / n)+\cos (2 l k \pi / n) \pm \sqrt{(\cos (2 l j \pi / n)-\cos (2 l k \pi / n))^{2}+1}, \quad l=0, \ldots, n-1 .
$$

Example 3.2. ([7, Corollary 2.4]) The eigenvalues of $R W(n, j, k)$ are

$\cos (2 l \pi / n)+\cos (2 l j \pi / n) \pm \sqrt{(\cos (2 l \pi / n)-\cos (2 l j \pi / n))^{2}+2+2 \cos (2 l k \pi / n)}, \quad l=0, \ldots, n-1$.

Example 3.3. ([7, Corollary 2.5]) The eigenvalues of $T(n, r, k, j)$ are

$$
\cos (2 l \pi / n)+\cos (2 l j \pi / n) \pm \sqrt{(\cos (2 l \pi / n)-\cos (2 l j \pi / n))^{2}+\alpha_{l}}, \quad l=0, \ldots, n-1,
$$

where $\alpha_{l}=3+2(\cos (2 \pi l r / n)+\cos (2 \pi l k / n)+\cos (2 \pi l(r-k) / n))$.

Since any Cayley graph over a group $G$ is a 1-Cayley graph over $G$, as a direct consequence of Theorem 3.3, we can reprove the following result which is proved in [27]:

Corollary 3.1. Let $\Gamma=\operatorname{Cay}(G, S)$ be a Cayley digraph over a finite group $G$ with irreducible matrix representations $\varrho^{(1)}, \ldots, \varrho^{(m)}$. Let $d_{l}$ be the degree of $\varrho^{(l)}$. For each $l \in\{1, \ldots, m\}$, define a $d_{l} \times d_{l}$ block matrix $A_{l}:=\left[A_{S}^{(l)}\right]$, where $A_{S}^{(l)}=$ $\sum_{s \in S} \varrho^{(l)}(s)$. Let $\chi_{A_{l}}(\lambda)$ and $\chi_{A}(\lambda)$ be the characteristic polynomial of $A_{l}$ and $A$, the adjacency matrix of $\Gamma$, respectively. Then $\chi_{A}(\lambda)=\Pi_{l=1}^{m} \chi_{A_{l}}(\lambda)^{d_{l}}$.

Let $G$ be a finite abelian group. Then by [16, Theorem 9.8], putting $n=2$ in Theorem 3.3, Theorem 3.2 directly follows. Also Theorems 4.6 and 4.3 of [12] improved in [1]:

Corollary 3.2. ([1, Corollary 9]) Let $\Gamma=\operatorname{Cay}(G, S)$ be a Cayley digraph, $H=\langle a\rangle$ a cyclic subgroup of $G$ of order $n$ and of index 2 with left transversal $\left\{t_{1}, t_{2}\right\}$. Then the characteristic polynomial of the adjacency matrix of $\Gamma$ is $\chi_{A}(\lambda)=\Pi_{k=0}^{n-1}(\lambda-$ $\left.\lambda_{k}^{+}\right)\left(\lambda-\lambda_{k}^{-}\right)$, where

$$
\begin{aligned}
& \lambda_{k}^{+}=\frac{\lambda_{k}^{11}+\lambda_{k}^{22}+\sqrt{\left(\lambda_{k}^{11}-\lambda_{k}^{22}\right)^{2}+4 \lambda_{k}^{12} \lambda_{k}^{21}}}{2}, \\
& \lambda_{k}^{-}=\frac{\lambda_{k}^{11}+\lambda_{k}^{22}-\sqrt{\left(\lambda_{k}^{11}-\lambda_{k}^{22}\right)^{2}+4 \lambda_{k}^{12} \lambda_{k}^{21}}}{2},
\end{aligned}
$$

$\lambda_{k}^{i j}=\sum_{t \in T_{j i}} \omega_{n}^{k t}$ and $T_{i j}=\left\{t \mid 0 \leq t \leq n-1, a^{t} \in t_{j} S t_{i}^{-1}\right\}$. 
Let $\Gamma$ be a $k$-regular graph with $n$ vertices and adjacency matrix $A$ and $A^{c}$ be the adjacency matrix of the complement of $\Gamma$. Then $(\lambda+k+1) \chi_{A^{c}}(\lambda)=$ $(-1)^{n}(\lambda-n+k+1) \chi_{A}(-\lambda-1)$, see $[9$, p. 20]. Despite of Cayley graphs, $n$-Cayley graphs $n \geq 2$, are not necessarily regular, but we have a similar relation between the characteristic polynomials of any $n$-Cayley graph and its complement which is given in the next theorem:

Theorem 3.4. ([1, Theorem 10]) Let $\Gamma=\operatorname{Cay}\left(G, T_{i j} \mid 1 \leq i, j \leq n\right)$ be an $n$ Cayley graph over a finite group $G, n \geq 1$. Let $\Gamma^{c}$ be the complement of $\Gamma$ with adjacency matrix $A^{c}$. Then the characteristic polynomials of $\Gamma$ and $\Gamma^{c}$ are related with the following equation:

$$
\chi_{B_{1}}(\lambda) \chi_{A}(-\lambda-1)=(-1)^{|G|-1} \chi_{A_{1}}(-\lambda-1) \chi_{A^{c}}(\lambda),
$$

where $B_{1}=|G| J-I_{n}-A_{1}, J$ is the all ones matrix of degree $n$, and $A_{1}=$ $\left[\left|T_{j i}\right|\right]_{1 \leq i, j \leq n}$.

An eigenvector of the adjacency matrix of a graph $\Gamma$ is said to be main eigenvector if it is not orthogonal to the all ones vector $\mathbf{j}$. An eigenvalue of a graph $\Gamma$ is said to be a main eigenvalue if it has a main eigenvector. By Perron-Frobenius Theorem, the largest eigenvalue of a graph is a main eigenvalue. It is also well known that a graph is regular if and only if it has exactly one main eigenvalue. So for every Cayley graph $\Gamma=\operatorname{Cay}(G, S),|S|$ is the only main eigenvalue of $\Gamma$. Since $n$-Cayley graphs, for $n \geq 2$ are not necessarily regular, determining the main eigenvalues of these graphs seems to be important. This problems reduced to determining main eigenvalues of the matrix $A_{1}$ :

Theorem 3.5. ([1, Corollary 12]) Let $\Gamma=\operatorname{Cay}\left(G, T_{i j} \mid 1 \leq i, j \leq n\right)$ be an $n$ Cayley graph over a finite group $G$ and $n \geq 2$. The main eigenvalues of $\Gamma$ is equal to main eigenvalues of the matrix $A_{1}=\left[\left|T_{j i}\right|\right]_{1 \leq i, j \leq n}$.

\section{Integrality of $n$-Cayley graphs}

A graph $\Gamma$ is called integral if all eigenvalues of the adjacency matrix of $\Gamma$ are integers. The concept of integral graphs was first defined by Harary and Schwenk [14]. During the last forty years many mathematicians have tried to construct and classify some special classes of integral graphs including Cayley graphs(for a survey see [21]). It seems that integral graphs are very rare and determining all the integral $n$-Cayley graphs, even for $n=2$, is difficult. It is easy to construct integral semi-Cayley graphs over finite abelian groups, as the following corollary shows:

Corollary 4.1. ([12, Corollary 3.5]) Let $\Gamma=\mathrm{SC}(G ; R, R, S)$ be a semi-Cayley graph over a finite abelian group $G$. If $\operatorname{Cay}(G, R)$ and $\operatorname{Cay}(G, S)$ are integral then $\Gamma$ is integral. 
The study of integrality of bi-Cayley graphs started by Arezoomand and Taeri in 2015:

Theorem 4.1. ([4, Corollary 3.10]) Every bi-Cayley graph of a finite group $G$ is integral if and only if $G$ is isomorphic to one of the groups $\mathbb{Z}_{2}^{k}, k \geq 1, \mathbb{Z}_{3}$ or $S_{3}$.

Also finite groups admitting a connected cubic integral bi-Cayley graph determined in the following theorem:

Theorem 4.2. ([8, Theorem A]) A finite group $G$ admits a connected cubic integral bi-Cayley graph if and only if $G$ is isomorphic to one of the groups

$$
\mathbb{Z}_{2}^{2}, \mathbb{Z}_{3}, \mathbb{Z}_{4}, \mathbb{Z}_{6}, \mathbb{Z}_{2} \times \mathbb{Z}_{6}, S_{3}, A_{4}, \text { Dic } c_{12} .
$$

The following questions naturally arise:

Problem 4.1. Determine finite groups admitting a connected $k$-regular, $k \geq 4$, bi-Cayley graphs.

Problem 4.2. Let $\Gamma=\operatorname{BCay}(G, S)$. In what conditions on $S$, is $\Gamma$ an integral?

Problem 4.3. Determine finite groups in which all bi-Cayley graphs over them of the valency at most $k \geq 2$ are integral.

Problem 4.4. Let $\Gamma=\operatorname{SC}(G ; R, L, S)$ be a semi-Cayley graph over a group $G$. In what conditions on $R, L$ and $S$ is $\Gamma$ an integral?

\section{Laplacian and signless Laplacian eigenvalues of $n$-Cayley graphs}

Let $\Gamma$ be a graph with vertex set $\left\{v_{1}, \ldots, v_{n}\right\}$. Recall that the adjacency matrix of $\Gamma$ is an $n \times n$ matrix $A=\left[a_{i j}\right]$, where $a_{i j}=1$ whenever $v_{i}$ and $v_{j}$ are adjacent and $a_{i j}=0$, otherwise. The degree matrix of $\Gamma$ is a diagonal $n \times n$ matrix $D=$ $\operatorname{diag}\left(d_{1}, \ldots, d_{n}\right)$, where $d_{i}$ is the number of vertices adjacent to $v_{i}$. The matrices $L=D-A$ and $Q=D+A$ are called Laplacian and signless Laplacian matrices of $\Gamma$, respectively. The characteristic polynomial of an $n \times n$ matrix $X$ is $\operatorname{det}\left(\lambda I_{n}-X\right)$, where $I_{n}$ is the $n \times n$ identity matrix and the roots of this polynomial are called eigenvalues of $X$. In this paper, the Laplacian eigenvalues and signless Laplacian eigenvalues of a graph $\Gamma$ are eigenvalues of Laplacian and signless Laplacian matrices of $\Gamma$, respectively.

In 2015, the Laplacian and signless Laplacian spectrum of semi-Cayley graphs over abelian groups computed:

Theorem 5.1. ([13, Theorem 1]) Let $\Gamma=\mathrm{SC}(G ; R, L, S)$ be a semi-Cayley graph over a finite abelian group $G=\mathbb{Z}_{n_{1}} \times \ldots \times \mathbb{Z}_{n_{t}}$. Then $\Gamma$ has Laplacian eigenvalues (resp. signless Laplacian eigenvalues)

$$
\frac{\mu_{r_{1} \ldots r_{t}}^{R}+\mu_{r_{1} \ldots r_{t}}^{L}+2|S| \pm \sqrt{\left(\mu_{r_{1} \ldots r_{t}}^{R}-\mu_{r_{1} \ldots r_{t}}^{L}\right)^{2}+4\left|\lambda_{r_{1} \ldots r_{t}}^{S}\right|^{2}}}{2}
$$


$r_{j}=0, \ldots, n_{j}-1, j=1, \ldots, t$, where $\lambda_{r_{1} \ldots r_{t}}^{S}$ are eigenvalues of Cay $(G, S)$, and $\mu_{r_{1} \ldots r_{t}}^{R}, \mu_{r_{1} \ldots r_{t}}^{L}$ are the Laplacian (resp. signless Laplacian) eigenvalues of $\mathrm{Cay}(G, R)$ and $\operatorname{Cay}(G, L)$, respectively.

The $n$-sunlet graph on $2 n$ vertices is obtained by attaching $n$ pendant edges to the cycle $C_{n}$. It is easy to see that $\Gamma=\operatorname{SC}(G, R, S, T)$, where $G=\langle a\rangle \cong \mathbb{Z}_{n}$, $R=\left\{a, a^{-1}\right\}, S=\varnothing$ and $T=\{1\}$.

Example 5.1. Let $\Gamma$ be an $n$-sunlet graph. Then

(1) Lpalcian eigenvalues of $\Gamma$ are

$$
2-\cos \frac{2 \pi l}{n} \pm \sqrt{\left(1-\cos \frac{2 \pi l}{n}\right)^{2}+1},
$$

where $l=0, \ldots, n-1$.

(2) signless Laplacian eigenvalues of $\Gamma$ are

$$
2+\cos \frac{2 \pi l}{n} \pm \sqrt{\left(1+\cos \frac{2 \pi l}{n}\right)^{2}+1}
$$

where $l=0, \ldots, n-1$.

As a corollary, one can construct semi-Cayley graphs with an integral Laplacian and signless Laplacian spectrum:

Corollary 5.1. ([13, Corollary 4.6]) Let $\Gamma=\mathrm{SC}(G ; R, R, S)$ be a semi-Cayley graph over a finite abelian group $G$. If $\mathrm{Cay}(G, R)$ and $\mathrm{Cay}(G, S)$ are integral graphs then $\Gamma$ is a Laplacian and signless Laplacian integral graph.

We end the paper with some open problems:

Problem 5.1. Determine the Laplacian and signless Laplacian eigenvalues of semiCayley graphs over non-abelian groups. Also do this for $n$-Cayley graphs when $n \geq 3$.

Problem 5.2. In what conditions on $R, L$ and $S, \operatorname{SC}(G ; R, L, S)$ is Laplacian (and signless Laplacian) an integral?

\section{R E F E R E N C E S}

1. M. Arezoomand and B. TAeri: On the characteristic polynomial of n-Cayley digraphs, Electron. J. Combin., 20(3) (2013), \# P57.

2. M. Arezoomand and B. TAeri: Normality of 2-Cayley digraphs, Discrete Math., 338 (2015), 41-47.

3. M. Arezoomand and B. TAeri: Isomorphisms of finite semi-Cayley graphs, Acta Math. Sin. Engl. Ser., 31(4) (2015) 715-730. 
4. M. Arezoomand and B. TAERI: A classification of finite groups with integral biCayley graphs, Trans. Combin., 4(4) (2015) 55-61.

5. M. Arezoomand and B. TAERI: Finite BCI-groups are solvable, Int. J. Group Theory, 5(2) (2016) 1-6.

6. M. AREzoOmAnd and B. TAERI: Which elements of a finite group are non-vanishing?, Bull. Iranian Math. Soc., 42(5) (2016) 1097-1106.

7. M. Arezoomand and A. R. Ashrafi: On the spectrum of 2-type bicirculants, Proceeding of 10th Graph Theory and Algebraic Combinatorics Conference, Yazd, Iran, 2017, pp. 10-13.

8. M. Arezoomand and B. TAERI: Finite groups admitting a connected cubic integral bi-Cayley graph, Alg. Struc. Appl., 5(2) (2018) 35-43.

9. N. Biggs: Algebraic Graph Theory, Cambridge University Press, 1974.

10. S.F. DU and D. MARUŠIČ:An infinite family of biprimitive semisymmetric graphs, J. Graph Theory 32 (1999) 217-228.

11. S.F. Du and D. MARUššc: Biprimitive graphs of smallest order, J. Algebraic Combin. 9 (1999) 151-156.

12. X. GAO and Y. LuO: The spectrum of semi-Cayley graphs over abelian groups, Linear Algebra Appl., 432 (2010) 2974-2983.

13. X. GAO, H. LÜ and Y. HAO: The Laplacian and signless Laplacian spectrum of semiCayley graphs over abelian groups, J. Appl. Math. Comput., 51 (1) (2016) 383-395.

14. F. HARARY and A. J. SChWEnK: Which graphs have integral spectra? in "Graphs and Combinatorics (Proc. Capital Conf., George Washington Univ., Washington, D.C., 1973)", Lecture Notes in Mathematics 406. Springer, Berlin, 1974, pp. 45-51.

15. A. Hujdurović, K. Kutnar and D. Marušič: On normality of $n$-Cayley graphs, Appl. Math. Comput., 332 (2018) 469-476.

16. G. JAMES and M. LiEBECK: Representations and characters of groups, Cambridge University Press, Second Edition, 2001.

17. I. Kovács, I. Malnič, D. Marušič, and Š. Miklavič: One-Matching bi-Cayley graphs over abelian groups, European J. Combin. 30 (2009) 602-616.

18. I. Kovacs, B. Kuzman, A. Malnič and S. Wilson: Characterization of edgetransitive 4-valent bicirculants, J. Graph Theory, DOI: 10.1002/jgt.20594

19. K. Kutnar, D. MARušič, Š. MiklaviČ and P. Šparl: Strongly regular tri-Cayley graphs, European J. Combin. 30(4) (2009) 822-832.

20. Z.P. LU, C.Q. WANG and M.Y. XU: Semisymmetric cubic graphs constructed from bi-Cayley graphs of $A_{n}$, Ars Combin. 80 (2006) 177-187.

21. X. Liu and S. Zhou: Eigenvalues of Cayley graphs: arXiv: 1809.09829 [math.CO].

22. K.H. Leung and S.L. MA: Partial difference triples, J. Algebraic Combin. 2 (1993) 397-409.

23. L. MARTinez: Strongly regular $m$-Cayley circulant graphs and digraphs: Ars Math. Contemp. 8 (2015) 195-213.

24. D. MARušič and D. MARUi: Strongly regular bicirculants and tricirculants, Ars Combin. 25 (1988) 11-15.

25. M. J. D. RESMi and D. JungniCKel: Strongly regular Semi-Cayley graphs, J.Algebraic Combin. 1 (1992), 171-195. 
26. G. SABIDussi: Vertex-transitive graphs, Monatsh. Math., 68 (1964) 426-438.

27. P. Diaconis and M. Shahshahani: Generating a random permutation with random transpositions, Z. Wahrsch. Verw. Gebiete, 57 (1981) 159-179.

28. J. X Zhou: Every finite group has a normal bi-Cayley graph, Ars Math. Contemp., 14 (2018) 177-186.

29. H. Zou and J.X. Meng: Some algebraic properties of Bi-Cayley graphs, Acta Math. Sin. (Chin. Ser.) 50 (5) (2007) 1075-1080.

Majid Arezoomand

Faculty of Engineeing

University of Larestan

74317-16137

Larestan, Iran

arezoomand@lar.ac.ir 